\title{
Improved Refractive Index Sensitivity Utilizing Long-Period Gratings with Periodic Corrugations on Cladding
}

\author{
Anthony Lim, Wen Bin Ji, and Swee Chuan Tjin \\ Photonics Research Centre, School of Electrical and Electronic Engineering, Nanyang Technological University, \\ 50 Nanyang Avenue, Singapore 639798 \\ Correspondence should be addressed to Anthony Lim, limc0074@e.ntu.edu.sg
}

Received 17 March 2012; Accepted 8 May 2012

Academic Editor: Yu-Lung Lo

Copyright ( $(2012$ Anthony Lim et al. This is an open access article distributed under the Creative Commons Attribution License, which permits unrestricted use, distribution, and reproduction in any medium, provided the original work is properly cited.

A new structure of Long-Period Gratings (LPGs) sensor is introduced as a sensitive ambient RI sensor. This structure consists of creating periodic corrugations on the cladding of the LPG. The experimental results show that this LPG structure has good performances in terms of linearity and sensitivity and serves as a highly sensitive and cost-effective sensor. It also has the advantage of portability as the corrugation can also serve as the reservoir for the specimen collection to be tested.

\section{Introduction}

In the last few years, Long-Period Gratings (LPGs) had been established as an effective sensor element for a wide range of external environmental measurement, ranging from strain, bending, and temperature to ambient Refractive Index (RI). Its small feature size, added onto the sensor high sensitivity, makes it an attractive sensor choice for miniaturization in technology trend [1]. As an ambient RI sensor, the low cost and ease of disposal offers LPG as a good sensing platform that can be utilized in many chemical and biological sensing applications [2]. Many papers based on different configurations of LPG had been published [3-7]. The standard LPGs are direct-written by UV exposure where the gratings couple light from the core mode to various cladding modes in the single mode fiber (SMF). As a result, a series of attenuation bands in the fiber transmission are created at discrete wavelength. This sensor works on the principle of changes in the attenuation bands with variation in the external environment parameters such as temperature, strain, and ambient refractive index. Recently, there are also several papers on improving the RI sensitivity: the chemical etch on the LPG cladding, tilted LPG, fabrication of complex structure and the deposition coating on LPG [8-13]. Another group of LPG sensors are formed with corrugations fabricated on its cladding. These corrugations are formed through either wet etch or $\mathrm{CO} 2$ laser ablation [14-17]. These sensors had been used in mainly strain sensing applications. As an ambient RI sensing, corrugation on SMF cladding had a sensitivity of $26.7 \mathrm{~nm} / \mathrm{RIU}$ using the wet etch in the imprint lithography [18].

LPG fabrication by the UV radiation is the most common method for gratings fabrication. The LPGs created in this study are created using this method. In the UV directwriting process, radiation from the UV laser is exposed onto the photosensitive fiber via an amplitude mask to create refractive index modulation on the core fiber. The amplitude mask consists of typical modulation depth of $10^{-4}$, period between $100 \mu \mathrm{m}$ to $500 \mu \mathrm{m}$ and length of $2 \mathrm{~cm}$ to $4 \mathrm{~cm}$. With the direct-writing process, the fiber's core index modulation is responsible for the light coupling from the propagating core mode to the copropagating cladding modes. With the copropagating cladding modes dissipated along the cladding of the fiber, the transmission spectrum consists of a series of attenuation bands centered at resonant wavelengths that depends on the effective index of the coupled modes and the gratings pitch. The phase matching between the core mode and the $i$ th forward-propagating cladding modes is achieved at the resonant wavelength and denoted in the following [19]:

$$
\lambda_{\text {res }}=\left(n_{\text {eff_co }}-n_{\text {eff_cl }}^{i}\right) \Lambda \text {, }
$$




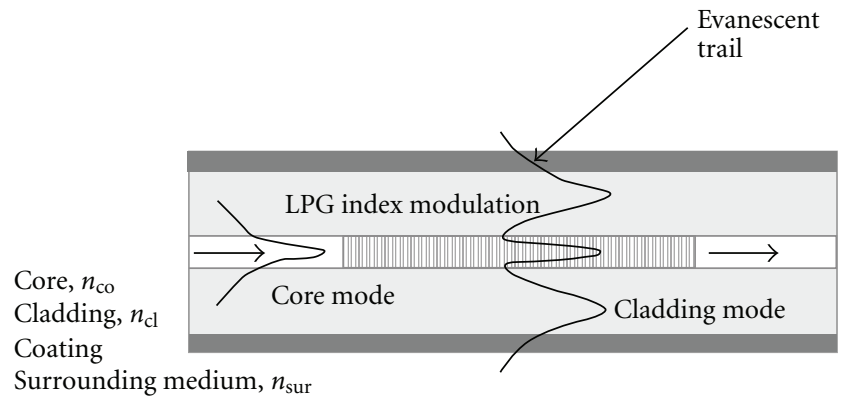

FIGURE 1: A schematic of the LPG which shows the core mode confined in the fiber core while the cladding modes extend into the cladding-air interface.
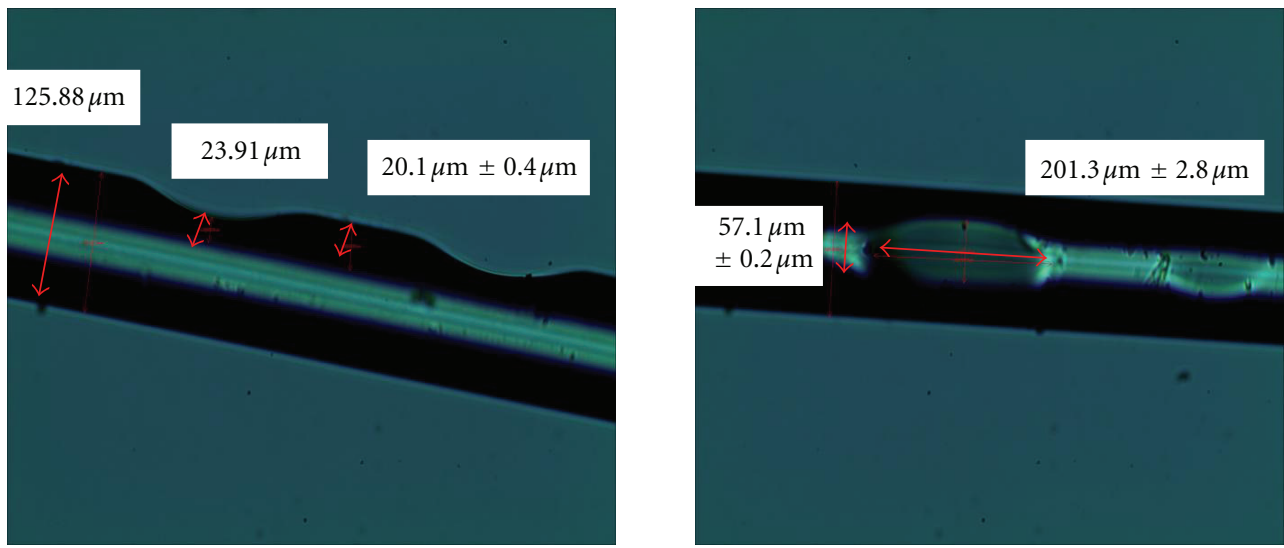

Figure 2: Measurement using the microscope: side and top views, respectively.

where $\lambda_{\text {res }}$ is the resonant wavelength, $\Lambda$ is the grating period, $n_{\text {eff_co }}$ and $n_{\text {eff_cl }}^{i}$ are the effective indices of the fundamental core mode and the $i$ th cladding mode, respectively.

As an ambient RI sensor, the variations in the ambient RI surrounding the cladding of an LPG can be expressed as [20]

$$
\frac{d \lambda}{d n_{\text {sur }}}=\frac{d \lambda}{d n_{m, \text { clad }}^{\text {eff }}} \frac{d n_{m, \text { clad }}^{\text {eff }}}{d n_{\text {sur }}}
$$

where $n_{\text {sur }}$ and $n_{m \text {,clad }}^{\text {eff }}$ are the refractive index of the surrounding material and ith effective cladding mode, respectively. The term $d n_{m \text {,clad }}^{\text {eff }}=d n_{\text {sur }}$ is distinct for each cladding mode, and hence an LPG depends strongly on the order of the coupled cladding modes. For a given LPG and cladding modes, the wavelength shift resulted from the ambient refractive index changes may be positive or negative depending on the local slope of the phase matching curve $d \lambda / d \Lambda$ [21]. As shown in Figure 1, the core mode is tightly confined in the fiber core while the cladding modes extend into the cladding-air interface which allows the evanescent wave to react with the ambient medium and results in its sensitivity to ambient RI change. This sensitivity to ambient refractive index changes can be either enhanced or suppressed by manipulating the fiber parameters [22]; the variations in the fiber radius will affect the cladding modes field distribution in the cladding-air interface which will change the optical power related to cladding modes evanescent wave in the external medium.

\section{Experimental Setup}

The LPG was direct-written on the Single Mode Fiber (SMF) core using the $248 \mathrm{~nm}$ Excimer laser (Lambda Physik, model Compex 205) via an amplitude mask. The amplitude mask has a specification of $320 \mathrm{um}$ period, $2.8 \mathrm{~cm}$ length. The $\mathrm{CO}_{2}$ laser (Epilog legend TT, model 7000) with 50\% speed and $30 \%$ power was next used to create periodic corrugations on the cladding of the LPG fibers. The $\mathrm{CO}_{2}$ laser has a maximum power of $40 \mathrm{~W}$ with maximum speed of $80 \mathrm{ips}$ (in per sec). With the ablation on the LPG cladding using 30\% power and $50 \%$ speed, this can be computed to about $12 \mathrm{~W}$ power and an estimate of constant speed of 40 ips (discarding the acceleration and deceleration). The total length of the corrugated cladding region is about $2.8 \mathrm{~cm}$ which is the length of the LPG. The corrugated fibers were inspected visually and measured with the microscope (CytroViVa). The fibers were then tested with different RI solutions, ranging from 1.3334 to 1.3807 that was prepared with different concentrations of salt in water. With the Stabilized Broadband Source (model 1RBL-11111-F), the wavelength responses to different RI solutions were measured with the Optical Spectrum Analyzer (Andro, model AQ6317). 

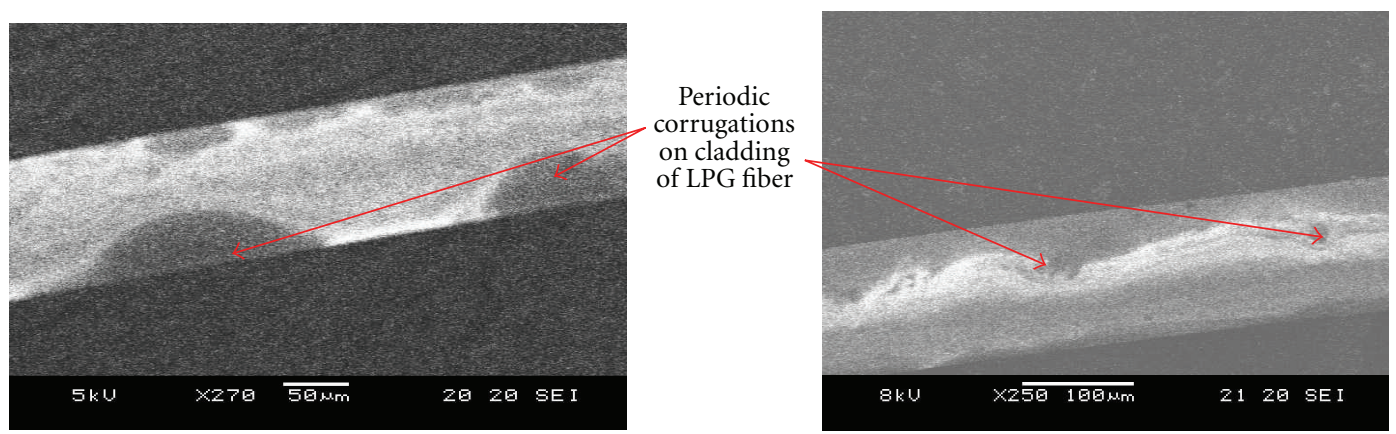

FIGURE 3: Using the SEM: side and top views, respectively.

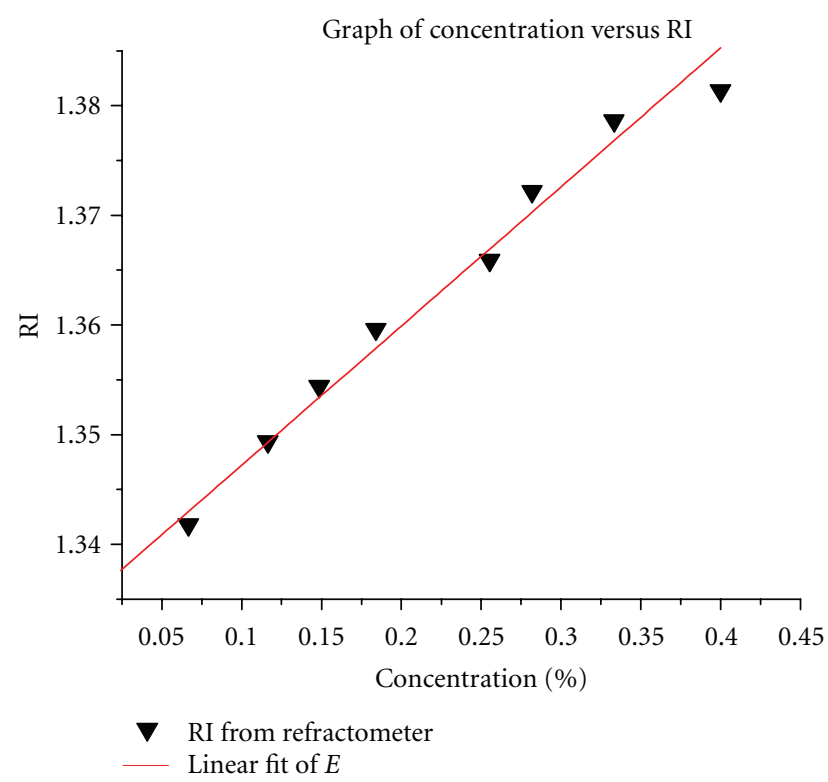

FIGURE 4: The correlation between different concentrations of salt solution with RI.

\section{Results and Discussion}

After the $\mathrm{CO}_{2}$ laser ablation on the LPG fibers' core, the fibers were measured with the microscope with the measurements highlighted in Figure 2. The periodic corrugation is elliptical in shape and measured with a dimension of $201.3 \mathrm{um} \pm$ $2.8 \mathrm{um}$ by $57.1 \mathrm{um} \pm 0.2 \mathrm{um}$ and with depth of $20.1 \mathrm{um}$ $\pm 0.4 \mathrm{um}$. The specimen collected in the corrugations is $23.91 \mathrm{um}$ from the core of the fiber. The period between two adjacent corrugations is about $281.7 \mathrm{um} \pm 3.4 \mathrm{um}$. Figure 3 shows the Scanning Electron Microscope (SEM) views.

Next different concentrations of salt were dissolved in water to obtain different RI solutions that were measured with a refractometer. The linear correlation is shown in Figure 4.

The corrugated LPG was tested with the different RI solutions and it was observed that the different cladding modes have different sensitivities [23]. The highest order attenuation band or resonant wavelength has the most sensitive wavelength shift to the ambient refractive index change. The wavelength responses to different RI solutions for the $1550 \mathrm{~nm}$ spectrum are shown in Figures 5 and 6 .
From Figure 7, the wavelength shifts were plotted and with the linear fit, the corrugated LPG gives a sensitivity of $611 \mathrm{~nm} / \mathrm{RIU}$ for the RI range from 1.3334 to 1.3807 . With the corrugations, the specimen are collected and measured at $23.91 \mathrm{um}$ from the core of the fiber. This increased the cladding modes field distribution at the cladding-air interface in the corrugations which accounts for the increased in the sensitivity to the ambient RI measured.

\section{Conclusion}

In this paper we have presented an in-fiber refractometer sensor that has periodic corrugations inscribed onto the cladding of an LPG. The corrugations improve the sensor sensitivity to ambient RI measurement while it simultaneously functions as a reservoir to collect and hold the specimen. In addition, this sensor can also be used to measure and identify different level of concentrations in specimens. The sensor has the advantages of high sensitivity, small feature size, compact, ease of use and offers potential for use in various chemical and biological sensing applications in the future. With the in-fiber corrugations that serve also as 


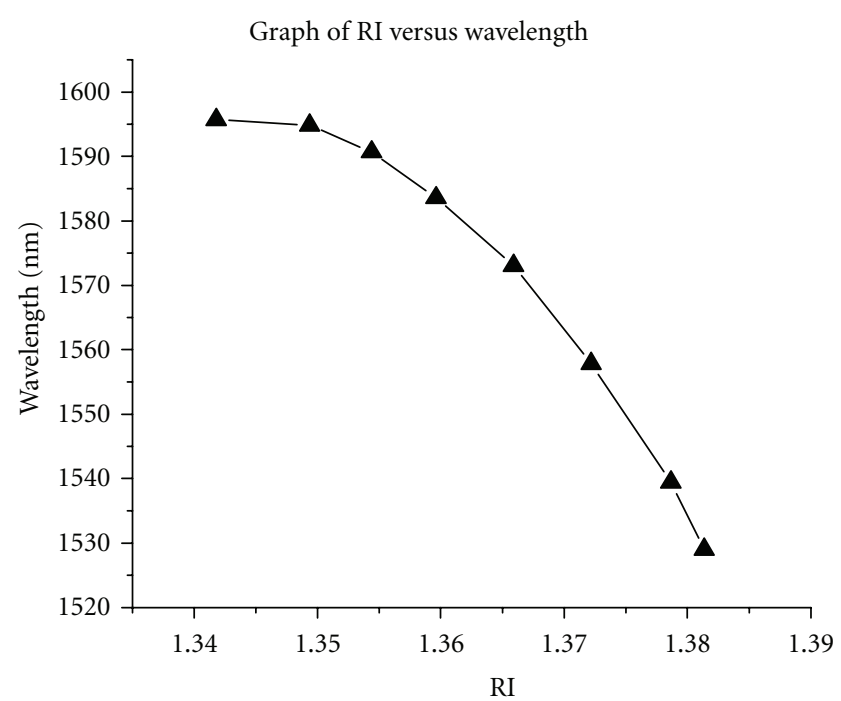

-^- Wavelength

Figure 5: The wavelength responses to different RI solutions.

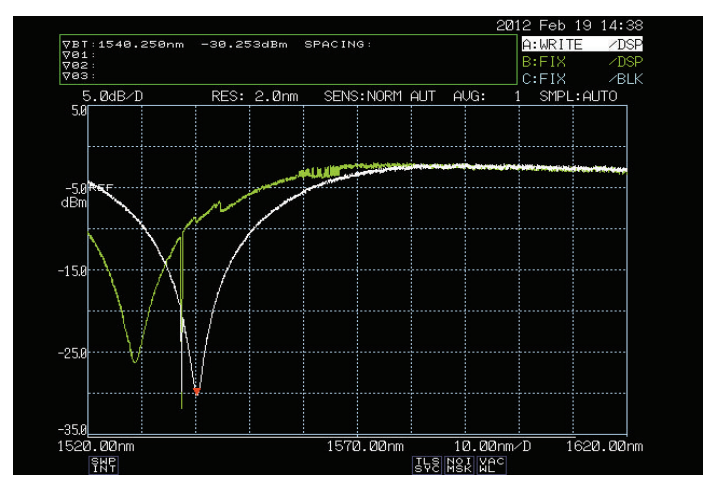

FIGURE 6: The spectral response curve to different RI solutions.

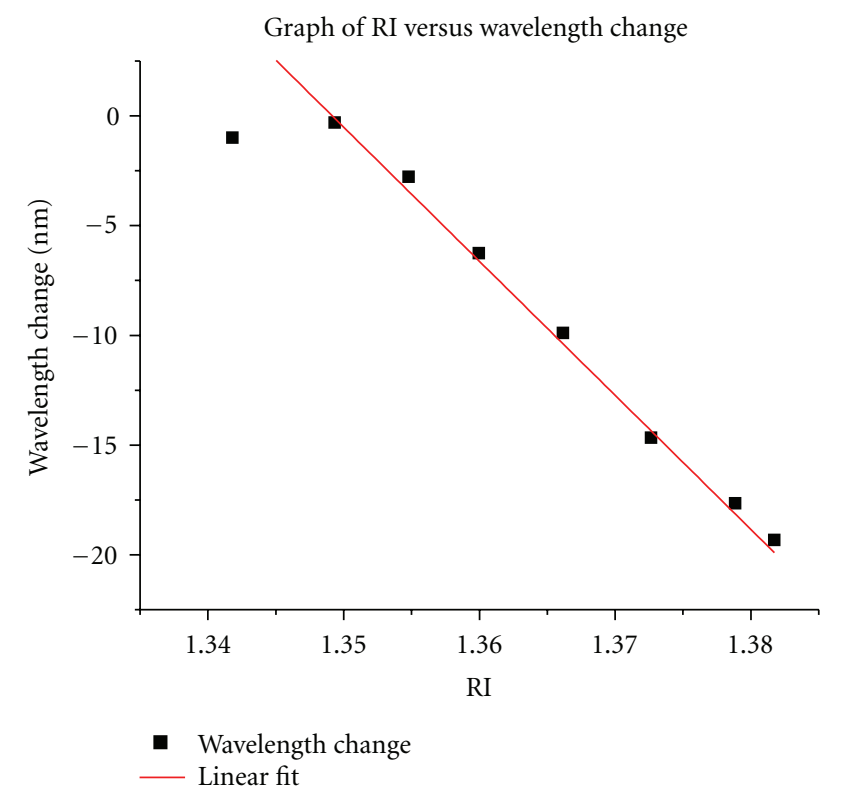

FIGURE 7: The wavelength change responses to different ambient RIs. 
the collection reservoir, it can also be deployed as an onsite sensor to collect specimen for measurement and disposed thereafter due to its low cost.

\section{References}

[1] Sensor Trends 2014, Trends in Future-Oriented Sensor Technologies, Association for Sensor Technology, Europe.

[2] A. Méndez, "MCH Engineering, LLC," in Proceedings of the the 14th Optoelectronics and Communication Conference, July 2009.

[3] Z. Tian, S. S. H. Yam, J. Barnes et al., "Refractive index sensing with Mach-Zehnder interferometer based on concatenating two single-mode fiber tapers," IEEE Photonics Technology Letters, vol. 20, no. 8, pp. 626-628, 2008.

[4] T. Zhu, Y. Fan, M. Deng, and Y. Rao, "Highly sensitive refractive index sensor based on two cascaded long period gratings with rotary refractive index modulation," in Optical Fiber Sensors, vol. 7753 of Proceedings of the SPIE, May 2011.

[5] J. Yang, L. Jiang, S. Wang, Q. Chen, B. Li, and H. Xiao, "Highly sensitive refractive index optical fiber sensors fabricated by a femtosecond laser," IEEE Photonics Journal, vol. 3, no. 6, Article ID 6084805, pp. 1189-1197, 2011.

[6] J. Kang, X. Dong, M. Li, Z. Zhang, and S. Jin, "Refraction index measurement using long period grating fabricated by symmetrical-hole fiber," in Passive Components and FiberBased Devices VII, vol. 7986 of Proceedings of SPIE, December 2010.

[7] M. S. Kwon and S. Y. Shin, "Refractive index sensitivity measurement of a long-period waveguide grating," IEEE Photonics Technology Letters, vol. 17, no. 9, pp. 1923-1925, 2005.

[8] J. Yan, A. P. Zhang, L. Y. Shao, J. F. Ding, and S. He, "Simultaneous measurement of refractive index and temperature by using dual long-period gratings with an etching process," IEEE Photonics Technology Letters, vol. 17, no. 11, 2005.

[9] A. Iadicicco, S. Campopiano, A. Cutolo, M. L. KorwinPawlowski, W. J. Bock, and A. Cusano, "Refractive index sensitivity in thinned UV and arc induced Long-Period Gratings: a comparative study," International Journal on Smart Sensing and Intelligent Systems, vol. 1, no. 2, 2008.

[10] E. Davies, P. Saffari, C. Mou, K. Zou, and L. Zhang, "Refractive index sensitivity enhancement of $81^{\circ}$ tilted Bragg gratings by cladding etching," in Optical Fibre Sensors, vol. 7503 of Proceedings of SPIE, October 2009.

[11] J. Yang, L. Yang, C. Q. Xu, and Y. Li, "Optimization of cladding-structure-modified long-period-grating refractiveindex sensors," Journal of Lightwave Technology, vol. 25, no. 1, pp. 372-380, 2007.

[12] E. Davies, R. Viitala, M. Salomäki, S. Areva, L. Zhang, and I. Bennion, "Sol-gel derived coating applied to long-period gratings for enhanced refractive index sensing properties," Journal of Optics A, vol. 11, no. 1, Article ID 015501, 2009.

[13] S. Korposh, S. W. Lee, S. W. James, and R. P. Tatam, "Refractive index sensitivity of fibre optic long period gratings with $\mathrm{SiO} 2$ nanoparticle based mesoporous coatings," in Optical Fiber Sensors, vol. 7753 of Proceedings of SPIE, May 2011.

[14] Y. P. Wang, W. Jin, and D. N. Wang, "Strain characteristics of CO2-laser-carved long period fiber gratings," IEEE Journal of Quantum Electronics, vol. 43, no. 2, pp. 101-108, 2007.

[15] C. C. Chiang, L. Tsai, H. J. Chang, C. L. Lin, and J. S. Kuo, "Fabrication of corrugated long-period fiber gratings by wet bulk micromachining," in Advanced Fabrication Technologies for Micro/Nano Optics and Photonics III, vol. 7591 of Proceedings of SPIE, January 2010.

[16] C. Y. Lin, L. A. Wang, and G. W. Chern, "Corrugated longperiod fiber gratings as strain, torsion, and bending sensors," Journal of Lightwave Technology, vol. 19, no. 8, pp. 1159-1168, 2001.

[17] H. Y. Wang, S. M. Chuo, and L. A. Wang, "Fabrication and simulation of corrugated long period microfiber gratings," in Optical Fiber Sensors, vol. 7753 of Proceedings of SPIE, p. 4, May 2011.

[18] C. Y. Huang, W. L. Chan, S. M. Chuo, J. H. Chang, L. L. Chen, and L. A. Wang, "Packaged symmetric/asymmetric corrugated long period fiber gratings for refractive index sensing applications," in Optical Fibre Sensors, vol. 7503 of Proceedings of SPIE, October 2009.

[19] X. Shu, L. Zhang, and I. Bennion, "Sensitivity characteristics of long-period fiber gratings," Journal of Lightwave Technology, vol. 20, no. 2, pp. 255-266, 2002.

[20] V. Bhatia, "Applications of long-period gratings to single and multi-parameter sensing," Optics Express, vol. 4, no. 11, pp. 457-466, 1999.

[21] H. J. Patrick, A. D. Kersey, F. Bucholtz, K. J. Ewing, J. B. Judkins, and A. M. Vengsarkar, "Chemical sensor based on long-period fiber grating response to index of refraction," in Proceedings of the Conference on Lasers and Electro-Optics (CLEO '97), pp. 420-421, May 1997.

[22] H. J. Patrick, A. D. Kersey, and F. Bucholtz, "Analysis of the response of long period fiber gratings to external index of refraction," Journal of Lightwave Technology, vol. 16, no. 9, pp. 1606-1612, 1998.

[23] T. Allsop, L. Zhang, and I. Bennion, "Detection of organic aromatic compounds in paraffin by a long-period fiber grating optical sensor with optimized sensitivity," Optics Communications, vol. 191, no. 3-6, pp. 181-190, 2001. 

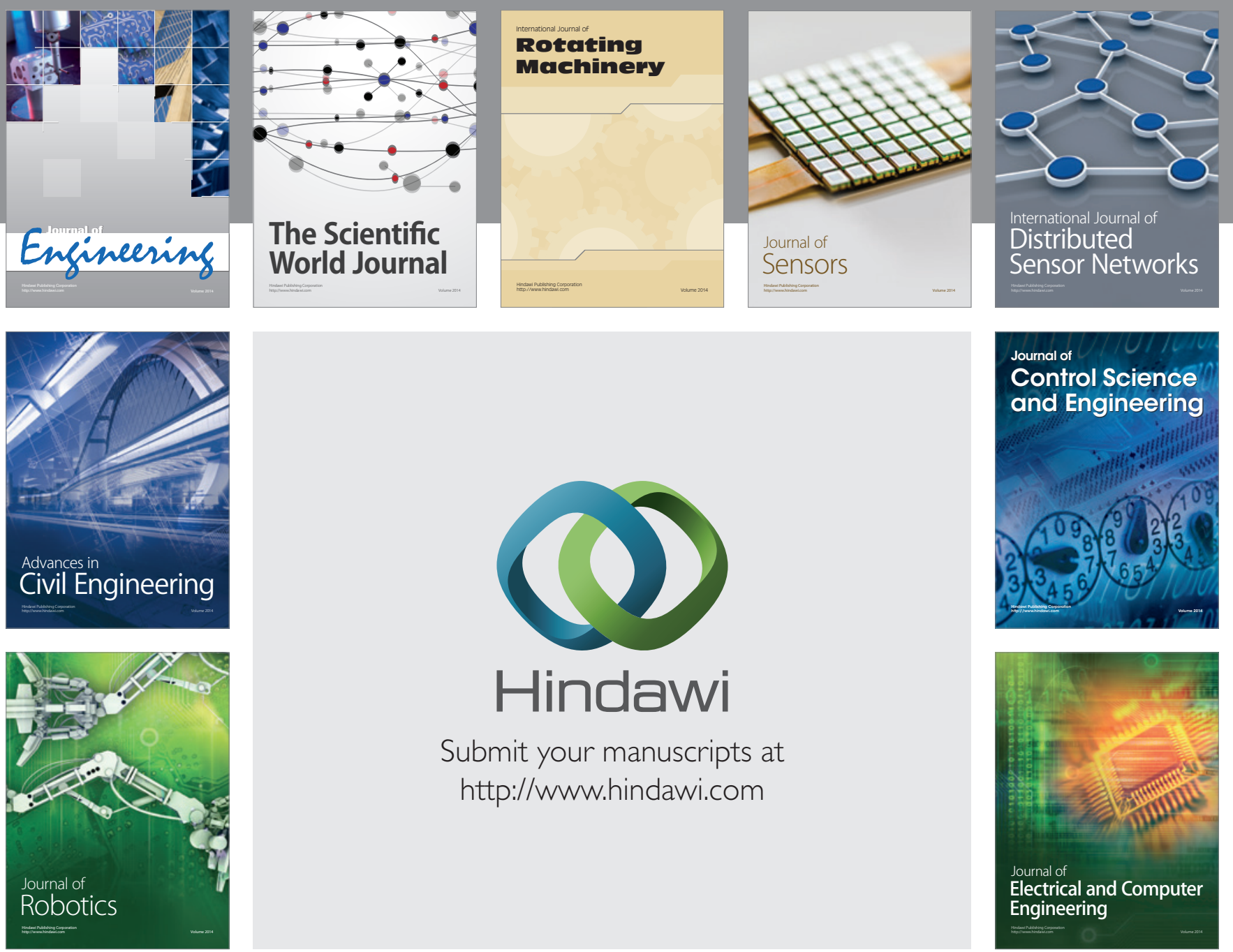

Submit your manuscripts at

http://www.hindawi.com
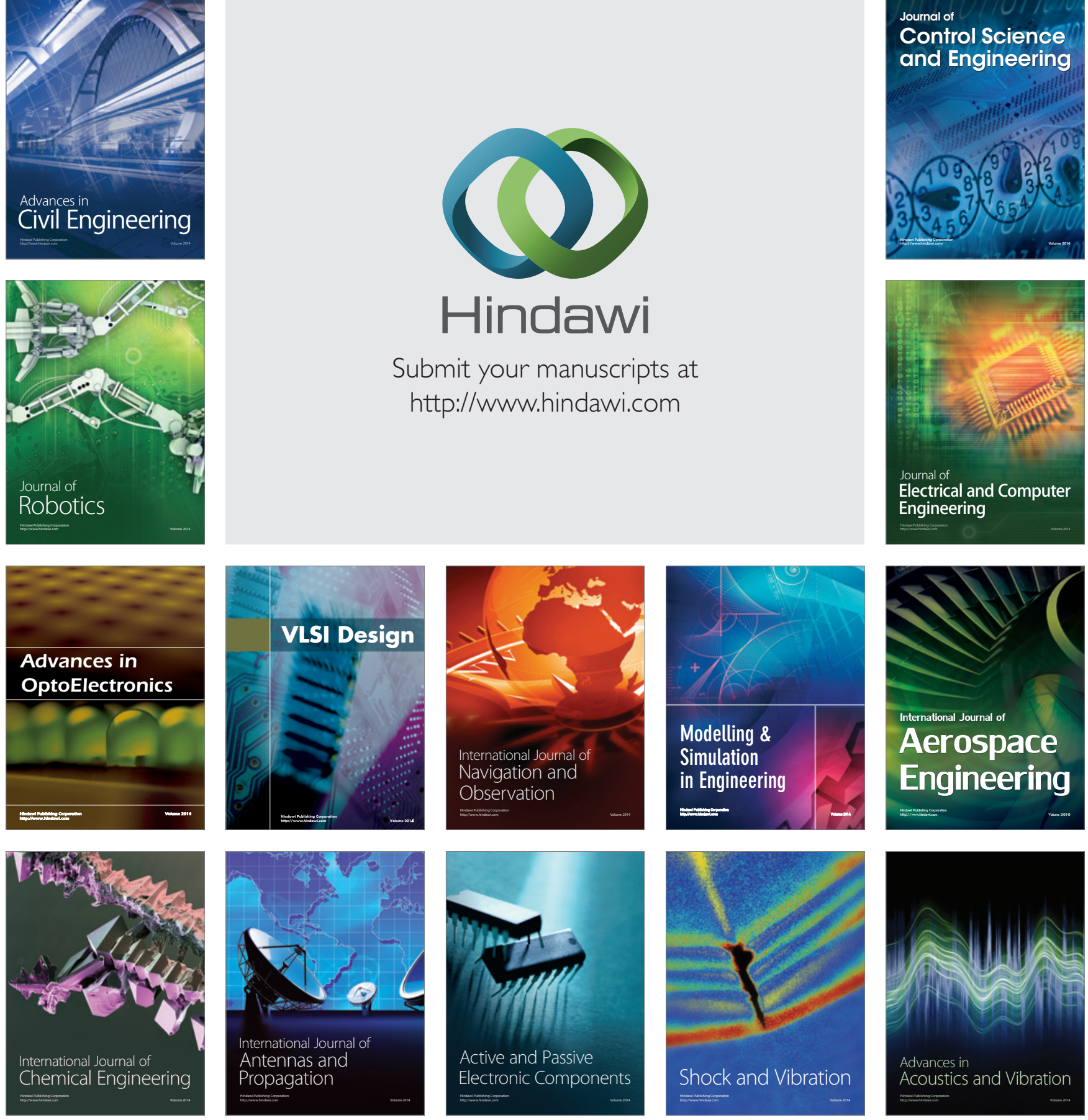